American Journal of Environmental Sciences 6 (3): 275-279, 2010

ISSN 1553-345X

(C) 2010 Science Publications

\title{
Residential Exposure of Environment Toxic Substance Outcome during Menstrual Cycle
}

\author{
${ }^{1,2}$ S. Alagendran, ${ }^{1}$ G. Archunan, ${ }^{3}$ M.P. Jonathan, ${ }^{2}$ E.A. Orozco-Bonilla, \\ ${ }^{4}$ Edgar Zentano Galindo and ${ }^{2}$ Rosalinda Guevara Guzman \\ ${ }^{1}$ Department of Animal Science, Centre for Pheromone Technology, \\ Bharathidasan University, Tiruchirapalli-24, Tamil Nadu, India \\ ${ }^{2}$ Sensorial Physiology Laboratory, Department of Physiology, \\ Faculty of Medicine, AV. Universidad 3000, CP, 04510, UNAM, Mexico \\ ${ }^{3}$ Centre for Investigation of Interdisciplinary Studies, IPN-Ciiemad, Mexico, DF \\ ${ }^{4}$ Department of Biochemistry, Faculty of Medicine, \\ AV. Universidad 3000, CP, 04510, UNAM, Mexico
}

\begin{abstract}
Problem statement: It was intended to diagnose and treat the disorders of female reproductive system where they are exposed to environmentally toxic pesticides and estrogenic compounds like bisphenol A which are the main reason for the infertility problems. Approach: Human saliva contains arrays of proteins that have distinct biological functions. Results: Salivary hormones concentrations of luteinising hormone, follicle stimulating hormone, estrogen as well as estrone-1-glucuronide, were assayed in 20 normal menstruating women who were among the reproductive phases like preovulatory phase (6-12 days); Ovulatory phase (13-14 days) and Postovulatory phase (15-26 days). It was intended to diagnose and treat the disorders of female reproductive system where they are exposed to environmentally toxic pesticides and estrogenic compounds like bisphenol A which are the main reason for the infertility problems. In this context, the detection of fertile period suggests that the mean value of luteinising hormone $\left(56.58 \pm 12.96 \mathrm{mIU} \mathrm{L}^{-1}\right)$ indicates the surge during the time of ovulation. Identifying the period of ovulation would be the easiest way to detect other constituents like follicle stimulating hormone, estrogen and its conjugate like estrone-1-glucuronide are extensively high $(\mathrm{p}<0.05)$ was observed. Conclusion: Our results indicated that the salivary hormones like estrogen and its conjugate indicates decrease in ovulatory and postovulatory phase. Further, the environmental toxic materials can also cause problems like infertility. The conclusion is that any physiological effects of estrogen from drinking water will be undetectable in people. The results from hormones suggested that they may be considered in the diagnosis and treatment of ovulation using saliva as the noninvasive specimen.
\end{abstract}

Key words: Menstrual cycle, estrogen, toxic chemicals, fertility problems

\section{INTRODUCTION}

The exact timing of ovulation in women is important to identify the fertile period. The fertility therapy like assisted reproduction is achieved is one of the reason as there will be environmental complications which also takes place in view of the pesticides resistance exposure and some chemicals in which (i.e., bisphenol A, DDT, Chloroethane, aldrin, dieldrin and eldrin) (Joffe and Li, 1994; Stoker et al., 1994). During the past decades many indirect tests have been conducted, which resulted in identifying the retrospective diagnosis of ovulation, but the prospective diagnosis of ovulation is important for management. Mean variations for hormonal parameters measured a precise menstrual cycle are complicated and there is considerable difference from cycle to cycle, even in the same patient (Joffe and $\mathrm{Li}, 1994)$. However, till date no method has achieved a $100 \%$ confidence regarding the impending of ovulation. Estrogens and progestins are naturally secreted in women as integral components of the menstrual cycle and excreted in urine as conjugated molecules. These conjugates are released as the natural stimulation of original steroids such as clomphine

Corresponding Author: G. Archunan, Department of Animal Science, Centre for Pheromone Technology, Bharathidasan University, Tiruchirapalli-24. Tamil Nadu, India 
citrate injection treatment and it tends to accelerate follicular development with multifollicular response and environmental complications which are premeditated both during the menstrual and non menstrual patients (Stoker et al., 1994). The major recent pharmacological addition to the natural steroid hormone excretion in women has been the oral contraceptives, particularly the synthetic estrogen ethinylestradiol, which is similarly excreted in the urine. Progesterone and related steroids are also of lower human potency than estrogen, though aquatic life may be more sensitive (Safe, 2000). The ovulation problems present themselves as irregular or anovulation and it can be substantiated through measurement of reproductive hormones. The environmental drawbacks come towards the menstrual patients is mainly due to the chemical exposure which causes toxicity in ovarian cycle. Transvaginal ultrasound and enzymatic estimation of LH has been proved as an extremely accurate tool for monitoring follicular development and ovulation, however, they are expensive and are not available in remote areas. Further, the present investigation indicates that the parameters like hormone in saliva are determined by means of menstrual cycle patients.

Several pesticides or their metabolites have been reported to possess estrogenicity in vivo (Gellert, 1978). Some of the pesticides are weakly estrogenic, but may act additively in combination of two or more pesticides. Further, when they are mixed together they induce estrogenic responses at concentrations lower than those required when each compound is administered alone (Stoker et al., 1994). Saliva is easy to collect via noninvasive techniques used by individuals with minimal exercise and can provide useful metabolic information (Lawrence, 2002). Historically, salivary analyses of female sex hormones were used for fertility monitoring (Garcia et al., 1981: Lipson and Ellison, 1996). However, recent findings indicate that these assays may be useful within the study of reproductive concerns. Estradiol, which was at high levels in women during their reproductive years, often causes increased immunoreactivity responsible for these phenomena (Gandara et al., 2007; Shirtcliff et al., 2001).

Many recent studies have been conducted to identify with the relationship between concentrations of various steroid hormones in saliva and plasma (Read et al., 1990), but there is discussion on the route by which steroids (or other hormones) enter saliva. In the blood only $1-15 \%$ of estradiol is in its unbound or biologically active form and the remaining estradiol is bound to serum proteins. The majority of estradiol in saliva is non-protein bound and enters the saliva via intracellular mechanisms (Hofman, 2001). We have examined the relation between saliva flow rate and salivary steroid concentration and between concentrations in serum and saliva. We believe the resulting insight into the mode of entry steroids and other hormones into saliva that will have important implications for the clinical utility of measuring these compounds in saliva.

\section{MATERIALS AND METHODS}

The studies were performed in 20 different healthy female adults from the age of 20-35 years. The volunteers were instructed to abstain from smoking; eating and drinking anything but water for $10 \mathrm{~h}$ prior to testing. In addition, all volunteers were asked to abstain from tooth brushing to prevent minimal gingival bleeding. The drinking water which was exposed to pesticides and chemicals used for cross examination during the normal menstrual subjects in triplicate manner.

Collection of samples: The saliva sample was collected from female volunteers during various periods viz., Preovulatory (6-12 days), ovulatory (13-14 days) and postovulatory phases (15-26 days). Whole saliva specimens were collected each morning for two consecutive menstrual cycles. Subjects were asked to spit the saliva into a $15 \mathrm{~mL}$ falcon tube for $10 \mathrm{~min}$ using tasteless paraffin wax for chew. The specimen was dated and stored in the subject's home freezer until the end of the second cycle, when it was picked up by research personnel and stored at $-20^{\circ} \mathrm{C}$ in the laboratory for up to 2-3 months until analysis (Hofman, 2001).

Extraction of samples: The sample was extracted by adding a preservative, sodium aside to keep it stable for longer than a week at room temperature. The samples were frozen immediate after collection. No samples are processed fresh; instead all were frozen and thawed before being divided into aliquots, so as to breakdown the mucopolysaccharides that can interfere with accurate pipetting. On the day of the assay, thaw the samples and was centrifuged $4000 \mathrm{rpm}$ for $10 \mathrm{~min}$ at $4^{\circ} \mathrm{C}$ are analyzed with immunoassay followed by commercially available kit by Saliva testing and Reference Laboratory (Seattle, WA, USA).

Statistical methods: The results are expressed as Mean \pm SEM. Those means in the same vertical column that are not marked with the same superscript letters and are significantly different at $p<0.05$. The relationship between the changes in hormone concentration was explored by means of one-way ANOVA, Posthoc-Dunnet test comparison between the phases of menstrual cycle using SPSS Software 
package version 5.0 (SPSS Inc, Chicago, USA). Significant $\mathrm{p}<0.05$ was only used for interpretation.

\section{RESULTS}

The environmentally toxic substances mainly involved during their reproductive cycle. The disease condition like follicular atresia or the ovulation failure was evaluated, where the follicular diameter showed a gradual increase in size (in $\mathrm{mm}$ ) followed by signs of ovulation (Fig. 1a) which, represents the mean concentration of the LH increases remarkably during the onset of preovulatory surge. During the maturation of the follicles the FSH varied drastically from cycle to cycle. The results indicate that, the value of FSH was concurrently increased from the 8th day (Fig. 1a). The fluctuation of estrogen is the best indicator for the identification of ovulation in female cycle and it can be distinguished from woman to woman during their menstrual cycle (Fig. $1 \mathrm{~b}$ and c). The estrogen conjugates like E-1-G indicate remarkable variation at the preovulatory phase of the menstrual cycle. The intraassay and interassay variation of the salivary hormone profile is comparable with the serum and urine values of E-1-G to assess the ovarian function of women. Figure 1a-c indicates the hormonal variation from phase to phase of menstrual cycle used to assess the ovulatory function. From the present investigation indicates the exposure of chemicals like pesticides such as DDT, bisphenol $\mathrm{A}$ and other toxic substances in drinking water would be great drawback; where hormones like LH, FSH and estrogen possess the adverse effects in women. Steroids, some other hormones and many drugs and antibodies can diffuse from serum into saliva, as a result of both passive diffusion and active transport, including ultrafiltration through tight cell junctions.

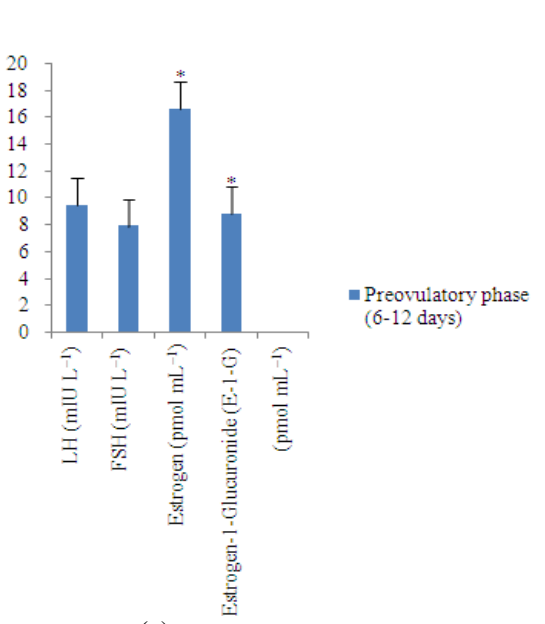

(a)

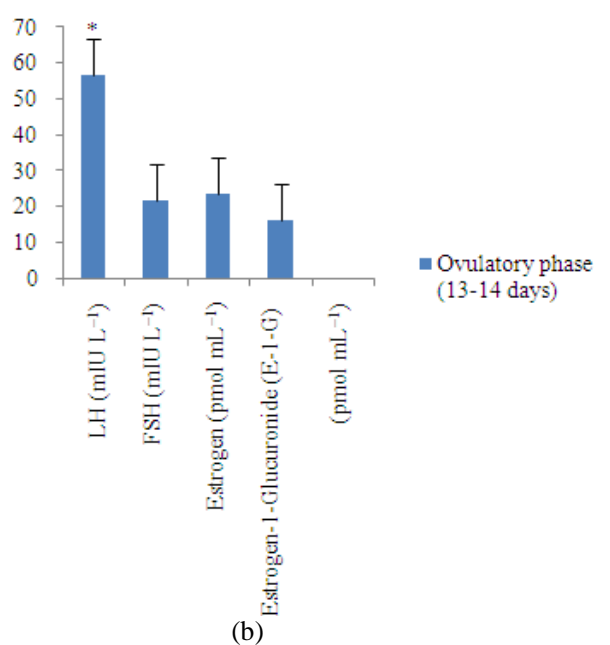

(b)

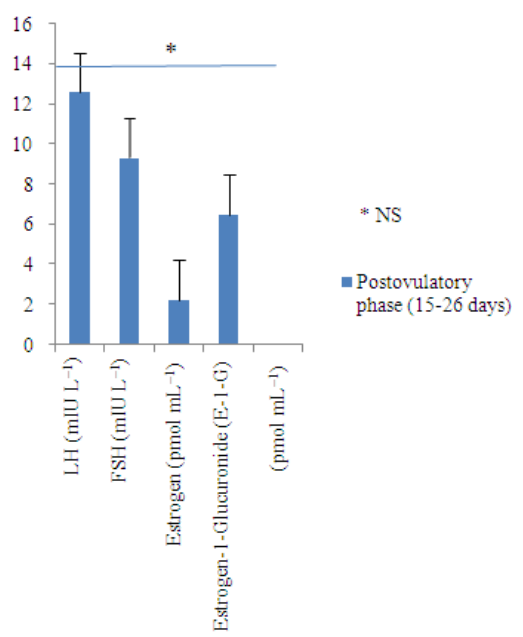

(c)

Fig. 1: Represent the salivary hormone variation in reproductive phases of menstrual cycle 
Am. J. Environ. Sci., 6 (3): 275-279, 2010

\section{DISCUSSION}

Many factors contribute to alter the hormone levels, which include both environmental and lifestyle induced factors (Goulet and Theriault, 1991). The altered hormone level may results from adaptive responses to a triggering factor, for example feedback control by the same or a related hormone, or from direct disturbance of correct hormonal control by an external agent like drug, pathogen and environmental factor such as pesticides containing components. The oral contraceptives that are currently in wide use contain ethinylestradiol, which is less easily metabolized than $17 \beta$-estradiol and hence requires lower doses to exert biological effects. The endocrine disruptors with estrogenic properties can possibly block ovulation similar to contraceptive pills. The midcycle surge of LH from the pituitary gland provides the physiological trigger for processing ovulation in women. Any chemical agent that compromises the LH surge could function as a reproductive toxicant (Goldman et al., 1991). In this study, low estrogen levels were found during anovulatory cycles. The pesticides thiram and sodium $\mathrm{N}$-methyldithiocarbamate also inhibit ovulation in rats.

A number of studies reported that women are occupationally exposed to pesticides in the agricultural sector have risks of spontaneous abortion (Arbuckle et al., 2001; Nurminen, 1995) and stillbirth (Goulet and Theriault, 1991; Pastore et al., 1997). Previous studies from pesticides contaminated water samples findings are consistent with studies of follicular development, which shows that a significant increase in circulating oestradiol and E-1-G concentrations occurs $\sim 1$ week before the LH surge.

The increase is associated with the appearance of the dominant follicle; the production of oestradiol is correlated with follicular surface area and large, oestrogen-rich follicles are the source of ova most likely to undergo successful fertilization and ongoing pregnancy. Thus, the higher follicular, particularly midfollicular, oestradiol concentrations we observed in conception cycles are associated with the selection and development, of dominant follicles with enhanced growth profiles and superior capacities to synthesize oestradiol (DiZercga and Hodgen, 1981). Pesticides contaminant drinking water causes the ovarian system in vivo in human and rats. To overcome this, Clomphine citrate injection plays an important role in the follicular development as well as for the production of estrogen during their fertile periods (Ellison et al., 1993; Markovic, 2001).
Our patients were healthy, well nourished, well educated women with no previous history of infertility and in remote areas it causes due to drinking water contaminated with pesticides are noteworthy. Thus they probably represent a relatively narrow sample of the total range of variability in female fecundity. Among preovulatory phases of women, we found no difference between LH concentrations until after the mid-luteal phase which considers the presumed time of implantation. In contrast, we found E-1-G a significant difference in comparison with oestradiol concentration during reproductive cycle, which was most pronounced during the mid-follicular phase, i.e. even before ovulation had occurred. Several pathways exist through which follicular oestradiol concentrations that may affect cycle fecundity. Earlier studies have shown that suppressed follicular development may lead to defective luteal function (Riad et al., 1980).

Our observation on correlation between relative body weight and mid-follicular oestradiol concentration supports the hypothesis of Belkien et al. (1985), that fertility is improved by $5-10 \%$ increases in body weight among women who practice weight control. However, Hoff (1983) have demonstration that periods of female weight loss were correlated with decreased ovulatory frequency. Ellison et al. (1993) have proposed that the sensitivity of ovarian function to energy balance provides means of adjusting female fecundity to ecological context. Recent studies have shown that insulin has a stimulatory effect on ovarian steroid production. On the other hand (Bates et al., 1982; Amot et al., 1995) suggest a mechanism by which weight gain could influence oestradiol concentrations. In the present study our results show that whole salivary samples collected at home by the women on a daily basis provide a noninvasive, cost effective and sufficient method of determining menstrual cycle profiles. Nevertheless, real-life occupational exposures to pesticides like DDT, bisphenol A, aldrin and eldrin (endocrine disruptors) appear to have adverse effects on female reproduction.

\section{CONCLUSION}

To find out targeted profiling to analyze saliva samples, following ultrafiltration to remove interfering proteins and particulate matter. The remaining small molecule metabolites can provide insight into serum composition that is otherwise difficult to obtain. Absorption of certain small molecule metabolites in saliva, including some hormones and drugs of abuse, are known to correlate quantifiably with concentrations in serum. Future research is needed on the ways in 
which pesticides may disrupt the hormonal function to analyze noninvasive, cost effective and cheap method.

\section{REFERENCES}

Amot, A.M., P. Vandekerckhove, M.A. DeBono and A.J. Rutherford, 1995. Follicular volume and number during in vitro fertilization' association with oocyte developmental capacity and pregnancy rate. Hum. Reprod., 10: 256-261.

Arbuckle, T.E., Z. Lin and L.S. Mery, 2001. An exploratory analysis of the effect of pesticide exposure on the risk of spontaneous abortion in an Ontario farm population. Environ. Health Perspect., 109: 851-857.

Bates, G.W., S.R. Bates and N.S. Whitworth, 1982. Reproductive failure in women who practice weight control. Fert. Ster., 37: 373-378.

Belkien, L.D., J. Bordt, P. Moller, R. Hano and E. Nieschlag, 1985. Estradiol in saliva for monitoring follicular stimulation in an in vitro fertilization program. Fert. Steril., 44: 322-327.

DiZercga, G.S. and G.D. Hodgen, 1981. Luteal phase dysfunction infertility a sequel to aberrant folliculogenes. Fert. Ster., 35: 489-499.

Ellison, P.T., C.P. Brick, S.F. Lipson and M.T. O'Rourke. 1993. The ecological context of human ovarian function. Hum. Reprod., 8: 2248-2258.

Gandara, B.K., L. Leresche and L. Mancl, 2007. Patterns of salivary estradiol and progesterone across the menstrual cycle. Ann. N. Y. Acad. Sci., 1098: 446-450.

Garcia, J.E., G.S. Jones and G.L. Wright Jr., 1981. Prediction of the time of ovulation. Fert. Ster., 36: 308-315.

Gellert, R.J., 1978. Kepone, mirex, dieldrin and aldrin: Estrogenic activity and the induction of persistent vaginal estrus and anovulation in rats following neonatal treatment. Environ. Res., 16: 131-138.

Goldman, J.M., R.L. Cooper, T.L. Edwards, G.L. Rehnberg, W.K. McElroy and J.F. Hein, 1991. Suppression of the luteinizing hormone surge by chlordime form in ovariectomized, steroid-primed female rats. Pharmacol. Toxicol., 68: 131-136.

Goulet, L. and G. Theriault, 1991. Stillbirth and chemical exposure of pregnant workers. Scand J. Work Environ. Health, 17: 25-31.
Hoff, J.D., M.E. Quigley and S.S.C. Yen, 1983. Hormonal dynamics at midcycle a reevaluation. J. Clin. Endocrinol. Metab., 57: 792-796.

Hofman, L.F., 2001. Human Saliva as a diagnostic specimen. J. Nutr., 131: 1621-1625.

Joffe, M. and Z. Li, 1994. Male and female factors in fertility. Am. J. Epidemiol., 140: 921-929.

Lawrence, H.P., 2002. Salivary markers of systemic disease: Noninvasive diagnosis of disease and monitoring of general health. J. Can. Dent. Assoc., 68: 170-174.

Lipson, S.F. and P.T. Ellison, 1996. Comparison of salivary steroid profiles in naturally occurring conception and non-conception cycles. Hum. Reprod., 11: 2090-2096.

Markovic, N., 2001. Women's oral health across the life span. Dent. Clin. North Am., 45: 513-521.

Nurminen, T., 1995. Maternal pesticide exposure and pregnancy outcome. J. Occup. Environ. Med., 37: 935-940.

Pastore, L.M., I.H. Picciotto and J.J. Beaumont, 1997. Risk of stillbirth from occupational and residential exposures. Occup. Environ. Med., 54: 511-518.

Read, G., R. Walker and D. Wilson, 1990. Steroid analysis in saliva for the assessment of endocrine function. Ann. N. Y. Acad. Sci., 595: 260-274.

Riad, F.D., G.F. Read and R.F. Walker, 1980. Salivary steroid assays for screening endocrine function. Postgrad. Med. J., 56: 75-78.

Safe, S.H., 2000. Endocrine disruptors and human health-is there a problem? An update. Environ. Health Perspect., 108: 487-493.

Shirtcliff, E.A., D.A. Granger, E. Schwartz and M.J. Curran, 2001. Use of salivary biomarkers in biobehavioral research: Cotton-based sample collection methods can interfere with salivary immunoassay results. Psychoneuroendocrinology, 26: $165-173$.

Stoker, T.E., R.L. Cooper, J.M. Goldman, J.E. Andrews and A.M.C. Soto et al., 1994. The pesticides endosulfan, toxaphene and dieldrin have estrogenic effects on human estrogen-sensitive cells. Environ. Health Perspect., 102: 380-383. 